\title{
Pathological practice and management of thyroid nodules: a Thai perspective
}

\author{
Nichthida Tangnuntachai ${ }^{1}$, Samreung Rangdaeng ${ }^{2}$, Supinda Koonmee ${ }^{3}$, Napadon Tangjaturonrasme ${ }^{4}$, \\ Somboon Keelawat ${ }^{1}$
}

${ }^{1}$ Department of Pathology, Faculty of Medicine, Chulalongkorn University, Bangkok, Thailand; ${ }^{2}$ Department of Pathology, Faculty of Medicine, Chiang Mai University, Chiang Mai, Thailand; ${ }^{3}$ Department of Pathology, Faculty of Medicine, Khon Kaen University, Khon Kaen, Thailand; ${ }^{4}$ Department of Otolaryngology, Faculty of Medicine, Chulalongkorn University, Bangkok, Thailand

Contributions: (I) Conception and design: N Tangnuntachai, N Tangjaturonrasme, S Keelawat; (II) Administrative support: N Tangnuntachai, N Tangjaturonrasme, S Keelawat; (III) Provision of study materials or patients: All authors; (IV) Collection and assembly of data: N Tangnuntachai, N Tangjaturonrasme, S Keelawat; (V) Data analysis and interpretation: N Tangnuntachai, N Tangjaturonrasme, S Keelawat; (VI) Manuscript writing: All authors; (VII) Final approval of manuscript: All authors.

Correspondence to: Napadon Tangjaturonrasme, MD; Somboon Keelawat, MD. Faculty of Medicine, Chulalongkorn University, Bangkok, Thailand. Email: Napadon.T@chula.ac.th; trcskl@gmail.com.

\begin{abstract}
Although, in the thyroid field, there have been several internationally standard diagnostic and management guidelines for practitioners from every country to follow but there is still variation among physicians across the globe in taking care of patients and pathology specimens with thyroid nodules due to dissimilar backgrounds of each place. Thailand is one among several countries that tries to maintain standard of care to meet international requirements although, with some limitations, not all recommendations are followed. To understand how approaches of thyroid nodules are performed throughout the country and how the healthcare system of the whole kingdom is managed including what major current limitations, the authors conducted a survey among physicians consisting of surgeons, radiologists and pathologists who are involved in the management of the thyroid lesions by personal phone contact and custom online questionnaires. Performances of fine needle aspiration of different institutes are also evaluated by analyzing the data from available publications and compared their results with other series from the literature. Shortage of pathologists and un-equal resource allocation are the major problems concerning administrative aspect while a lack of awareness in new terminologies or diagnostic systems (e.g., NIFTP, UMP, Bethesda, TI-RADS) among physicians, particularly clinicians, are the problems of training and continuous medical education. Another point of concern is a rather poor performance in fine needle aspiration from the Thai series in which there are high rate $(30.5 \%)$ of non-diagnostic category (Bethesda I) and false negativity as demonstrated by relatively high risk of malignancy (9.6\%) in Bethesda II compared with other series from the literature. Regarding molecular studies, despite their usefulness in improving diagnostic accuracy, especially for cytology specimens, these ancillary tools are not routinely used in our practice since they are available in only few referral centers. This review provided a background information of the perspective on how thyroid nodules are managed in the Thai settings.
\end{abstract}

Keywords: Thailand; Thai perspective; thyroid nodules; management; pathological practice

Submitted Mar 24, 2020. Accepted for publication Jun 05, 2020.

doi: $10.21037 /$ gs-20-395

View this article at: http://dx.doi.org/10.21037/gs-20-395 


\section{Introduction}

The practice of medicine in Thailand fully follows the international standard guidelines mostly developed by Western medical experts. However, the context between Thailand and Western societies are different, and so implementing these guidelines may not be suitable for a Thai setting and, therefore, they are modified. Such differences include the ratio of pathologists and differences in the healthcare reimbursement system. Within the thyroid field, there are several diagnostic systems and clinical guidelines that have been developed by Western professionals, such as the Bethesda System for Reporting Thyroid Cytopathology (TBSRTC) (1), the World Health Organization (WHO) classification of tumors on endocrine organs (2), the College of American Pathologists (CAP) protocol (3), the American Joint Committee on Cancer (AJCC) (4), the National Comprehensive Cancer Network (NCCN) (5), and the American Thyroid Association (6), etc. However, when these systems/guidelines are integrated into local Asian practices, there may be some outcomes from the local settings that are different from Western societies.

Thyroid carcinoma is one of the top 10 cancer sites in Thai women (7). This article elaborates on the clinical and pathological practices of managing patients with thyroid lesions in different medical centers in Thailand by conducting a survey among physicians who commonly deal with thyroid nodule patients. Personal contact by phone or custom online questionnaire was used and a total of 88 physicians from 51 Thai hospitals provided their perspectives in dealing with thyroid nodules. The details of the respondents are shown in Table 1. All of the authors have verified, interpreted, and discussed the data.

\section{National guidelines for management of thyroid nodules in Thailand}

As previously mentioned, the context of medical practice in a Thai setting may not be the same as that in the rest of the world, particularly compared to Western countries, and so the national guidelines for management of several diseases have been developed in Thailand as references for local practices, including the guidelines for the management of thyroid tumors (8), which has been published and distributed to different medical centers throughout the country since 2015.

The Thai national guidelines book was initiated and funded by the National Cancer Institute of Thailand. The working group consisted of 26 health professionals who were representatives of different hospitals throughout the country and were members of professional organizations, such as the Royal College of Surgeons of Thailand, Royal College of Otolaryngologists-Head and Neck Surgeons of Thailand, Royal College of Pathologists of Thailand (RCPath-T), the Endocrine Society of Thailand, Nuclear Medicine Society of Thailand, Thai Society of Clinical Oncology, Thai Association of Radiation Oncology, Association of Surgical Oncologist (Thailand), and the Institute of Medical Research and Technology Assessment. In addition to these members, there were also specialists from different fields that were involved in preparing the contents of this book. These content experts were from the fields of endocrinology $(n=4)$, nuclear medicine $(n=5)$, otolaryngology $(n=12)$, surgery $(n=2)$, oncology $(n=3)$, radiotherapy $(n=3)$, and pathology $(n=6)$. Finally, the draft was sent to nine experts of related disciplines for peer review before it was published.

Although there were over 150 references cited in this book, the core contents were mainly adopted from the NCCN (regarding clinical aspects) (5), and the WHO classification of tumors on endocrine organs (2), TBSRTC (1) and CAP protocol (3) (regarding pathological aspects), which were modified to fit the real practice conditions in Thailand. Recommendations were categorized into the four levels of 1 , $2 \mathrm{~A}, 2 \mathrm{~B}$, and 3 based on the strength of evidence and level of agreement among the experts (Table 2).

\section{Diagnostic approaches and management of patients with thyroid nodules in different settings in Thailand}

Most physicians use the American Thyroid Association guidelines followed by the guidelines developed by Thailand National Cancer Institute and the NCCN guidelines to manage cases with thyroid nodules. Patients who have a thyroid nodule can directly visit many physicians, ranging from general practitioners to specialists. Specialists who are involved in the management of the thyroid lesions include otolaryngologists, general surgeons, endocrine surgeons, head and neck surgeons, and endocrinologist.

The first step in managing thyroid nodules begins by obtaining the patient's medical history. Patients who have a rapidly growing mass, history of neck irradiation, familial history of papillary thyroid carcinoma or medullary thyroid carcinoma, and hoarseness of the voice with invasive symptom require special attention since these factors indicate that the patient may have cancer. 
Table 1 Demographic data of the respondents

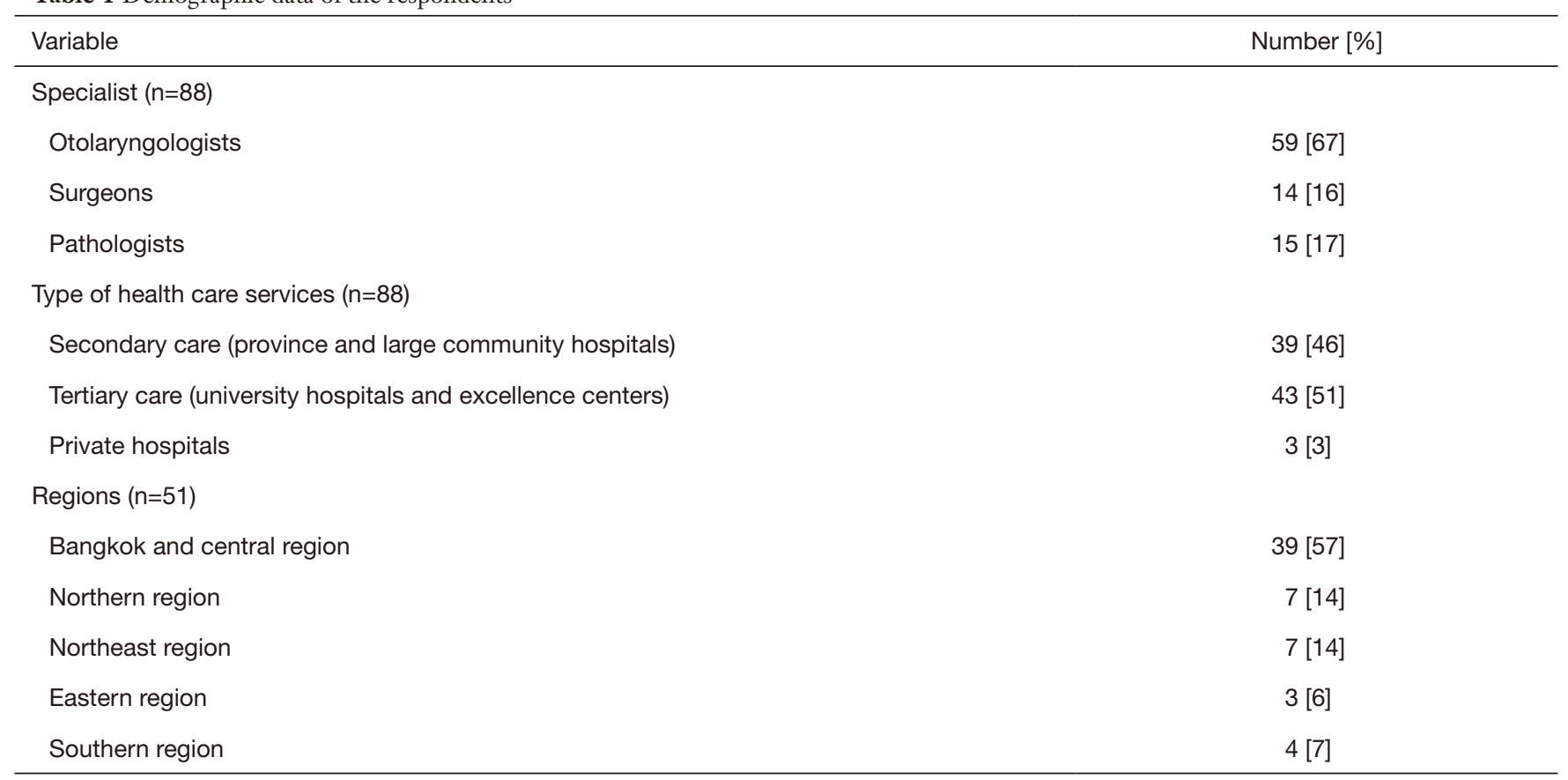

Table 2 Levels of recommendations from the National guidelines for the management of thyroid nodules in Thailand based on the strength of evidences and agreement among experts

\begin{tabular}{lcc}
\hline Categories of recommendation & Strength of evidence & Agreement among experts \\
\hline 1 & High reliability & Consensus \\
$2 \mathrm{~A}$ & Low reliability & Consensus \\
$2 \mathrm{~B}$ & Low reliability & No consensus \\
3 & No reliability & Very high disagreement \\
\hline
\end{tabular}

*, systematic review from randomized controlled trial or well-designed randomized controlled trial.

Then a physical examination is performed, where the characteristics and location of the nodule are recorded. Cervical lymphadenopathy and vocal cord paralysis are considered as very high-risk findings. In general, the assessment of vocal cord function will be done only by an otolaryngologist. However, since there are limited numbers of otolaryngologists available, physicians from other fields will rely only on the history of unchanged voice that is considered to be the normal vocal cord movement. Any sign or symptom of obvious functional abnormality (hypo-/ hyper-thyroidism) are usually noted.

In a large-scale hospital, there are currently around 10 20 new cases of patients with a thyroid nodule per physician per month. The proportion of patients whose thyroid nodule(s), without a palpable neck mass, were accidentally found during their health check-up by ultrasound screening are increasing.

Even without obvious signs and symptoms of a hormonal imbalance, more than $75 \%$ of the physicians request a blood test to assess the thyroid function to screen for subclinical diseases. This reflects the fact that tests for triiodothyronine free triiodothyronine, free thyroxine, thyroxine and thyroid stimulating hormone are readily available nationwide at a reasonable price and with an acceptable turn-around time. Screening for thyroid tumor markers, such as thyroglobulin, calcitonin, and carcinoembryonic antigen, is not commonly performed because of its low accuracy and because the incidence of medullary thyroid carcinoma is low in this region, except for the patients that have a familial history of this type of cancer. 
Currently, ultrasonography (USG) and fine needle aspiration (FNA) are the two main types of tests that are important for thyroid nodule work up. Many international guidelines use the sonographic criteria to evaluate the risk level of each nodule and tailor the treatment and followup according to the protocol recommendation. The USG is usually performed by diagnostic radiologists, where a routine evaluation focuses only on the thyroid gland without assessing the cervical lymph node. With the limited number of diagnostic radiologists in Thailand, an appointment for the procedure takes a long time and may even be unavailable in some areas. Occasionally, office-based sonography may be performed by surgeons who have experience in sonography, but this is used solely in the context of ultrasound-guided biopsy or neck surveillance. Most importantly, they do not have the authorization to sign official radiology reports.

The American College of Radiology (ACR)'s 2017 Thyroid Imaging Reporting and Data System's (TI-RADS) white paper provides another decision-assisting protocol in reference to the use of FNA and recommendations for follow-up visits. Due to its only recently being launched, this report system is not recognized by most Thai practitioners and has only been implemented in academic centers or some private hospitals. More than half of the surgeons are not familiar with the system and are reluctant to interpret this format of report (Figure 1).

Regardless, the FNA provides another key step in approaching thyroid nodules. Nowadays, all international guidelines recommend performing FNA only in cases with a high-risk USG finding, which is still not possible for routine practice in Thailand. The long waiting time to get an appointment for an USG assessment and the lack of interventional radiologists are the key limitations.

Most cytology specimens are prepared with conventional smears and fixed in $95 \%$ alcohol. Liquid-based cytology and interpretation of the results are done by experienced cytopathologists. However, at present, this can only be performed in large university hospitals. According to our survey, only $11 \%$ of public hospitals use a liquid-based preparation alone and a further $11 \%$ use a liquid-based preparation along with conventional staining.

Some hospitals have to outsource their pathology services to private laboratories. Cytopathology reports are usually available in 1 week ( $87 \%$ of respondents). However, due to the logistics of transporting the specimens and reports, the results are usually delivered to the respective hospital in 2 weeks, which still meets the target performance index set by the Ministry of Public Health of Thailand.
Almost all FNA (88-92\%) cases are reported using the current TBSRTC guideline 2017 (second edition) (1). In contrast, only $55 \%$ of the physicians are familiar and confident in interpreting the results using this system. This reflects the good pathology training curriculum and continuous educational programs for pathologists in Thailand, yet this reporting format is not recognized by many clinicians because they have not been trained to use this type of reporting system. This makes them reluctant to use it to guide them in making decisions as how to manage their patients.

Analysis of cytopathological reports in Thailand using the TBSRTC from 5 series (9-12) which included King Chulalongkorn Memorial hospital, Bangkok $(n=2,762)$, Chiang Mai University, Chiang Mai $(\mathrm{n}=3,143)$, Srinagarind Hospital, Khon Kaen ( $\mathrm{n}=701)$, Theptarin Hospital, Bangkok $(\mathrm{n}=2,735)$ and Rajavithi Hospital, Bangkok $(\mathrm{n}=1,371)$ were mostly concordant with studies from other countries $(1,13-15)$. However, the Thai series have a much higher Bethesda I category (mean 30.5\%) compared to others series as shown in Table 3 and Figure 2. Regarding the risk of malignancy (ROM) in each category (13), the Thai series showed a rather high ROM (average 9.6\%) in the Bethesda II, whereas the ROM in other series was lower (0-8\%) (1,13-16) as summarized in Table 3 and Figure 2.

Molecular studies and immunocytochemistry are not included in the routine FNA unless the lesions are cytologically unclear. Molecular studies are only available in academic hospitals and some of the tests may not be reimbursed under the universal healthcare service budget.

Universal grossing protocol for a solitary encapsulated thyroid nodule is based on submission guidelines (17). If the thyroid nodule is less than or equal to $2 \mathrm{~cm}$ in size, entire capsular sections are submitted for histological examination. Whereas, if the thyroid nodule is more than $2 \mathrm{~cm}$, at least 10 cassettes of suspected extracapsular extensions are submitted.

Patients who have an abnormal thyroid function should be managed medically by an endocrinologist. Patients with thyroid nodule with euthyroid and have no risk feature from the USG analysis may undergo thyroid hormonal suppression for 1-2 years. During suppression, the size of the nodule volume may decrease up to $50 \%$ from its original size. However, the nodules usually regrow after withdrawal of the suppressive treatment.

Surgical management depends on the indication for surgery. Common indications include large nodules, compressive symptoms, and suspected or proven malignancies. At present, there is no practice in Thailand for thyroid nodule enucleation. Thyroid lobectomy for unilateral 
A

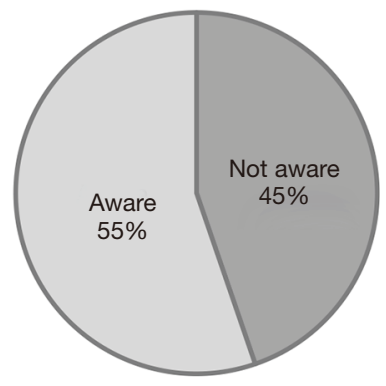

C

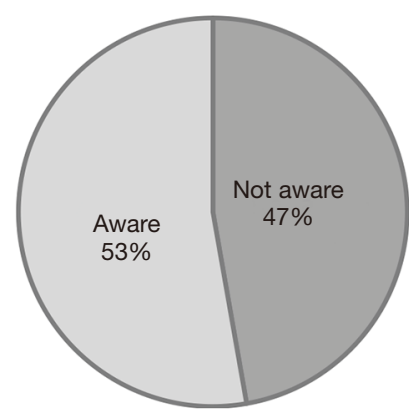

$\mathrm{E}$

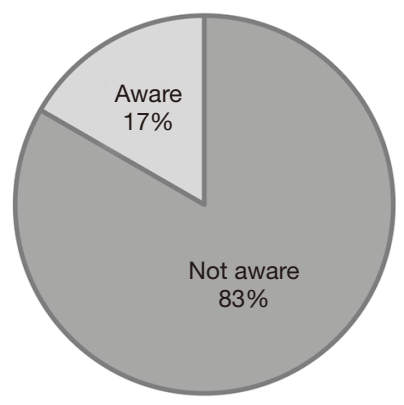

G

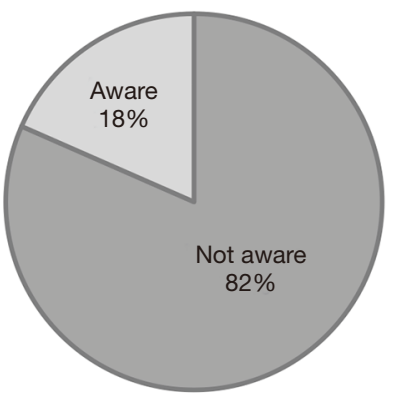

B

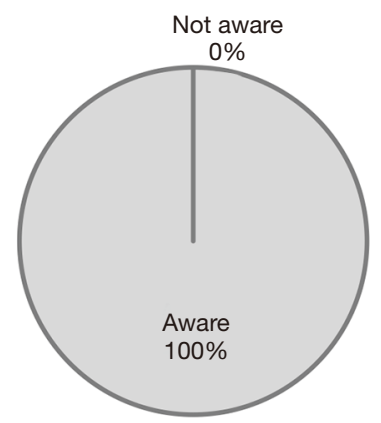

D

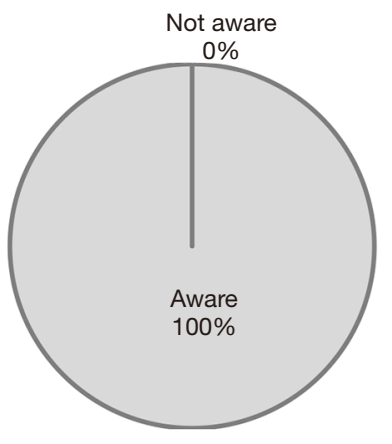

$\mathrm{F}$

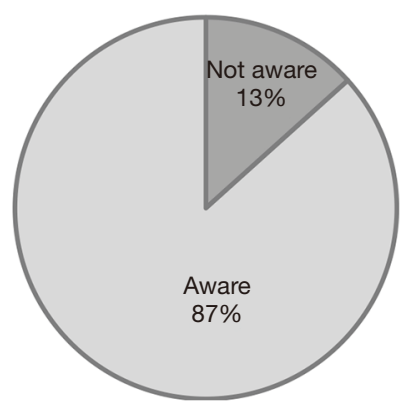

$\mathrm{H}$

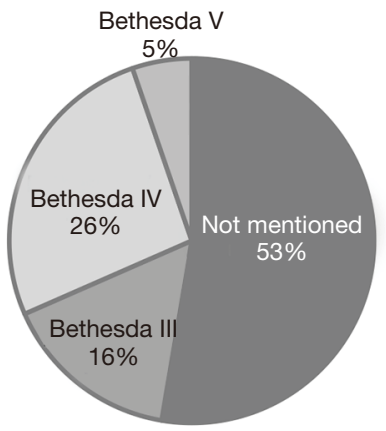

Figure 1 Awareness in new terminologies/reporting systems among clinicians and pathologists, shown as pie graphs displaying the awareness of interpreting reports using TBSRTC between the clinicians (A) and pathologists (B); (C,D) diagnosis of NIFTP between the clinicians (C) and pathologists (D); (E,F) diagnosis of UMP between the clinicians (E) and pathologists (F); $(\mathrm{G})$ the clinicians' awareness of the TIRADS system; and $(\mathrm{H})$ the percentage of Bethesda categories in which the term "NIFTP" was mentioned. 
Table 3 Frequency and malignancy rates of the Bethesda categories by cyto-histological correlation from Thai series compared to other studies

\begin{tabular}{|c|c|c|c|c|c|c|}
\hline FNA Bethesda category & \multicolumn{6}{|c|}{ Proportion (\%) } \\
\hline Frequency & $30.5(16.3-47.6)$ & 12.9 & 13.0 & NA & 12.2 & NA \\
\hline Frequency & $51.8(34.7-68.1)$ & 59.3 & 59.8 & NA & 62.3 & NA \\
\hline Risk of malignancy & $9.6(2.8-19)$ & 3.7 & 6.5 & 5.0 & $0.7-8$ & $0-3$ \\
\hline \multicolumn{7}{|l|}{ AUS/FLUS } \\
\hline Frequency & $6.8(2.4-21.7)$ & 10.1 & 11.0 & NA & 6.1 & NA \\
\hline Risk of malignancy & $35.7(6.1-63)$ & 26.1 & 33.1 & 25.0 & 28.9 & $25-40$ \\
\hline \multicolumn{7}{|l|}{ Suspicious for malignancy } \\
\hline Frequency & $3.1(1.4-5.0)$ & 2.7 & 3.7 & NA & 3.7 & NA \\
\hline Risk of malignancy & 77.7 (57-92.9) & 75.2 & 65.0 & 72.0 & 79.6 & $50-75$ \\
\hline \multicolumn{7}{|l|}{ Malignancy } \\
\hline Frequency & $9.1(3.2-22.9)$ & 5.4 & 6.0 & NA & 7.4 & NA \\
\hline
\end{tabular}

NA, not available.

lesions, and subtotal, near total, or total thyroidectomy for bilateral lesions may be chosen. Some surgeons may not perform a unilateral thyroid lobectomy even when established to be thyroid cancer due to limitation of the facility to perform proper post-operative follow-up care and long-term surveillance. The average waiting time for surgery is up to 6 months, and longer in large hospitals where there are more complicated cases. The approach for thyroid lobectomy can be the classical method or remote access surgery, such as the transoral endoscopic thyroidectomy vestibular (18), retroauricular $(19,20)$, or axillary-breast approaches (21), depending on the discussion between the surgeon's preference and the patient's needs.

For thyroid nodules, nuclear medicine is used (I) to evaluate the nodule function or treatment of hyperthyroidism, and (II) to treat patients with well differentiated thyroid cancers who have a positive indication for post-operative radioiodine therapy. External beam radiation is rarely used but may be indicated in patients who have an advanced stage thyroid cancer. Nuclear physicians and radiation oncologists are only available in some regional cancer centers or largescale hospitals. In Thailand, the current healthcare system divides the hospitals according to their geographic locations and groups them into various health clusters. In every cluster, there must be at least one center where nuclear medicine, radiation oncologist staff, and facilities are available. With this system, the government can provide standard treatment to the people through referral and cooperation between the clustered hospitals.

Pathological diagnosis of a thyroidectomy specimen is reported using Cancer Protocol Templates from the CAP guideline (3). Most cases are reported within 2 weeks after the specimens are submitted to the laboratories. However, due to limited number of pathologists and endocrine pathologists, very few cases are read by experts in the thyroid field. Immunohistochemistry may also 

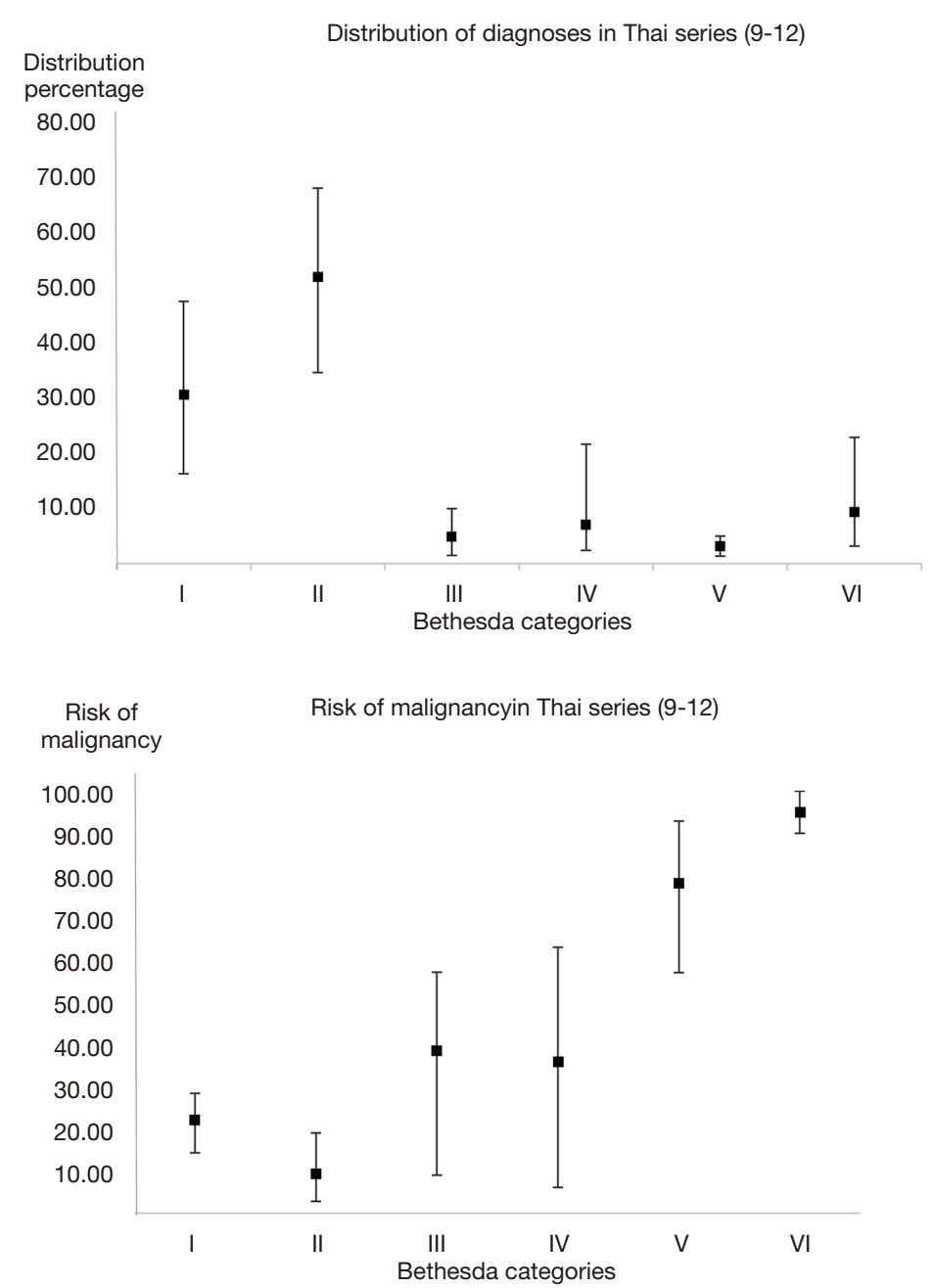

Figure 2 Distribution of diagnoses and risk of malignancy in Bethesda categories from the 5 Thai series (9-12).

be implemented by some pathologists in cases with an uncertain histology for diagnosis, such as examination of the expression patterns of cytokeratin 19, galectin-3, and Hector Battifora mesothelial-1. However, they are available only in some laboratories. If the laboratory does not have the facility to perform immunohistochemical studies, they can send their paraffin blocks to the National Referral Pathology Laboratory, the Institute of Pathology, Ministry of Public Health of Thailand. Most of the tests in Thailand are sent to the National Referral Pathology Laboratory. Once the staining is done, the immunohistochemical stained slides will be digitally scanned with a Whole Slide Imaging scanner. Pathologists who use this service can view the virtual slides by logging-in to the website of the Institute. This way, there is no need to wait for the slides to return to the lab to view the immunohistochemical studies.
Since not all treatment alternatives are available throughout Thailand, some cases are referred to a tertiary care hospital for treatment, in which previous pathology reports are reviewed by other pathologists. When there are discrepancies in the reports, a review panel or additional pathological investigations, such as immunohistochemistry are needed. However, the feedback loop is not well structured so the results are usually not given back to the former pathologists for review.

Currently, digital pathology is only used in a few private laboratories, where some of their pathologists are not able to work in the sites. Whereas, for public hospitals, this platform is used mainly for academic and research purposes rather than for services. In general, most pathologists prefer glass slides to digital images; they are more comfortable and confident in working with the glass slides. 
All laboratories are encouraged to be accredited by the RCPath-T and ISO 15189/15190. An external quality assessment program is available and provided by pathology societies in Thailand, such as the RCPath-T, International Academy of Pathology, Thailand Division, and Thai Society of Cytology.

\section{Awareness of new terminologies}

The diagnosis of thyroid malignancy in Thailand is reported using the WHO Classification of Tumors of Endocrine Organs, $4^{\text {th }}$ edition [2017] (2) and the CAP guidelines (3). There have been new terminologies that most clinicians are not aware of, such as Well Differentiated Tumor of Uncertain Malignant Potential (WDT-UMP), Follicular Tumor of Uncertain Malignant Potential (FT-UMP), and Noninvasive Follicular Thyroid Neoplasm with Papillary-like Nuclear Features (NIFTP). According to our survey, most clinicians were not familiar with these terminologies, especially the WDT-UMP and FT-UMP, with only $17 \%$ of clinicians having heard of these terminologies, whereas $87 \%$ of general pathologists were familiar with these entities (Figure 1). This may lead to inappropriate treatment. Only some pathologists mentioned NIFTP in their reports and most of these cases are classified as Bethesda III-IV (Figure 1).

\section{Limitations and improvements}

In 2010, the WHO examined and reported Thailand as one of the top 50 healthcare systems in the world and the second-best in Southeast Asia (22). Despite such high recognition and a successful universal health care system that has benefitted all citizens for almost 20 years, especially those with financial problems (22), there are still many issues that need to be solved. One of the most important obstacles is the inequality of access to healthcare and services. There are differences in management standards between medical centers at different levels in Thailand due to the availability of equipment and medical personnel.

There is a long queue for surgery, which is the largest and most serious problem when it comes to taking care of patients because it can delay treatment which may, in turn, negatively impact on the prognosis of the disease. According to our survey, the waiting time in most public hospitals was $2-6$ months.

There is a shortage of clinical specialists, as already mentioned. As a result of this, the waiting period to receive specific medical services may take a long time to acquire. The current training systems are slowly helping to increase the number of trainees each year. However, this may need some time before the changes become evident.

A lack of pathologists is also currently one of the biggest issues in Thailand. According to the RCPath-T, there are currently approximately 500 registered pathologists, of which 100 have retired. Therefore, for the 69.8 million people in Thailand, the ratio of active pathologists (about 400) per 100,000 -population is about 0.57 . This number is much lower (around 7- to 8.8-fold) than those of developed countries (e.g., the United States of America and Canada, etc.) where the ratio of pathologists per 100,000-population is as high as 4-5 (23). Moreover, there are many hospitals in remote areas where there are no pathologists. Thus, these hospitals have to seek pathology services from private laboratories. Most of these private laboratories are operated by part-time pathologists who already work full-time in government hospitals. Therefore, overall, most Thai pathologists are handling a large workload volume, which, as a result, may affect the quality of their reports, as well as their own health. In addition, under these conditions, there is also the extra-time needed to travel between the hospitals and the laboratories, which can delay the delivery of the laboratory results.

Each year, there are 45 slots available for anatomic pathology residency training throughout the country, but the slots were never filled up. One of the reasons why pathology is not a popular field among Thai medical graduates is because of low incentives. Another reason is that it is unrecognized by the Thai population. The RCPath-T has tried its best to resolve these problems by encouraging pathology laboratories to raise pathologists' fees/salaries and publicizing pathology career to the Thai medical graduates through several campaigns. Although the situation has improved recently, it is still not yet completely resolved.

With the low number of pathologists, and even lower numbers of experts in the thyroid specialty, the pathology service for thyroid lesions may not meet the expected excellent standard. Although Thailand has very active pathology societies, such as the RCPath-T, the International Academy of Pathology, Thailand Division and the Thai Society of Cytology, all of whom have regularly organized academic activities for many years, not all of the pathologists who take care of cytopathology and surgical pathology specimens of thyroid lesions can catch up with the rapidly growing knowledge in this field. Therefore, new terminologies of thyroid lesions (e.g., WDT-UMP and FTUMP) are unknown and not used by many pathologists and, as a consequence, are also unrecognized by clinicians.

Another problem in the management of thyroid nodules 
is that the cytology samples are often inadequately collected, which results in a non-diagnostic cytology report (Bethesda I). This is quite a common issue in the training centers because the trainees who usually take care of the FNA lack the skill in performing this procedure. In our survey, 1,317 out of 2,762 cytology samples of thyroid nodules (47.7\%) collected from King Chulalongkorn Memorial Hospital, Bangkok, Thailand during 2010-2015 were categorized as "Non-diagnostic/Unsatisfactory (Bethesda I)" (10). Upon reviewing the 5 series of thyroid cytology in different medical centers in Thailand, the average Bethesda I category was $30.5 \%$ (9-12). Rapid onsite evaluation by cytopathologists may provide a solution for the issue but this service is available only in a few centers.

Availability of molecular studies is another issue that causes inequality of standards among medical centers. Only a few centers, mostly university-based, can provide these tests (e.g., BRAF, TERT, RET, PAX-8/PPAR-G, and RAS) and most of them are rather expensive. Therefore, unless there is a need for targeted therapy, only a few cases may require these tests for diagnosis of thyroid cancer.

To improve the care of patients with thyroid nodules, different approaches have to be implemented by the institutes, organizations, and the government. The government needs to be involved in the distribution of medical personnel and provide the financial support to them. The institutes and professional societies must provide the most updated knowledge and facilitate the health care team to improve the necessary skills for patients' care.

\section{Conclusions}

This review provided a perspective on how thyroid nodules are managed in the Thai settings. Various aspects from guidelines used for diagnosis and management, real practice in different settings, awareness of new terminologies to limitations and improvements were discussed. We found that a shortage of medical personnel, especially pathologists, and unequal resource allocation seem to be the most important obstacles that need to be overcome. Other problems that also require correction include unawareness to updated knowledges on new pathological terminologies among practitioners, especially clinicians, and poor performance in fine needle aspiration cytology.

\section{Acknowledgments}

The authors thank all of the respondents who answered the questionnaires and so made it possible to complete this study on the management of thyroid nodules in Thailand, and also to Miss June Ohata from Office of Research Affairs, Faculty of Medicine and Prof. Robert Douglas from Office of Research Affairs, Chulalongkorn University for proofing the English language in this article.

Funding: None.

\section{Footnote}

Provenance and Peer Review: This article was commissioned by the Guest Editor (Kennichi Kakudo) for the series "Asian and Western Practice in Thyroid Pathology: Similarities and Differences" published in Gland Surgery. The article was sent for external peer review organized by the Guest Editor and the editorial office.

Peer Review File: Available at http://dx.doi.org/10.21037/gs20-395

Conflicts of Interest: All authors have completed the ICMJE uniform disclosure form (available at http:// dx.doi.org/10.21037/gs-20-395). The series "Asian and Western Practice in Thyroid Pathology: Similarities and Differences" was commissioned by the editorial office without any funding or sponsorship. The authors have no other conflicts of interest to declare.

Ethical Statement: The authors are accountable for all aspects of the work in ensuring that questions related to the accuracy or integrity of any part of the work are appropriately investigated and resolved.

Open Access Statement: This is an Open Access article distributed in accordance with the Creative Commons Attribution-NonCommercial-NoDerivs 4.0 International License (CC BY-NC-ND 4.0), which permits the noncommercial replication and distribution of the article with the strict proviso that no changes or edits are made and the original work is properly cited (including links to both the formal publication through the relevant DOI and the license). See: https://creativecommons.org/licenses/by-nc-nd/4.0/.

\section{References}

1. Ali SZ, Cibas ES. The Bethesda System for Reporting Thyroid Cytopathology: Definitions, Criteria, and Explanatory Notes. 2nd ed. Springer; 2018. 
2. Lloyd RV, Osamura RY, Klöppel G, et al. WHO Classification of Tumours of Endocrine Organs. 4th ed. Bosman FT, Jaffe ES, Lakhani SR, Ohgaki H, editors. Lyon, France: IARC Press; 2017.

3. Protocol for the Examination of Specimens From Patients With Carcinomas of the Thyroid Gland. 2019 [cited 10 January 20202]. Available online: https://documents.cap. org/protocols/cp-endocrine-thyroid-19-4200.pdf

4. Amin MB, Edge S, Greene F, et al. AJCC Cancer Staging Manual. 8th ed. Springer; 2017.

5. National Comprehensive Cancer Network. Thyroid cancer (Version 2.2019). Available online: https://www. nccn.org/professionals/physician_gls/pdf/thyroid.pdf

6. Haugen BR, Alexander EK, Bible KC, et al. 2015 American Thyroid Association Management Guidelines for Adult Patients with Thyroid Nodules and Differentiated Thyroid Cancer: The American Thyroid Association Guidelines Task Force on Thyroid Nodules and Differentiated Thyroid Cancer. Thyroid 2016;26:1-133.

7. Tangjaturonrasme N, Vatanasapt P, Bychkov A. Epidemiology of head and neck cancer in Thailand. Asia Pac J Clin Oncol 2018;14:16-22.

8. Chindavijak S, Panchan V, Chaiwerawattana A, et al. Guidelines for the diagnosis and treatment of thyroid cancer. Bangkok: Kosit Press Co., Ltd.; 2015.

9. Keelawat S, Rangdaeng S, Koonmee S, et al. Current Status of Thyroid Fine-Needle Aspiration Practice in Thailand. J Pathol Transl Med 2017;51:565-70.

10. Limlunjakorn P, Keelawat S, Bychkov A. Evaluation of thyroid fine needle aspiration cytology by the Bethesda reporting system: a retrospective analysis of rates and outcomes from the King Chulalongkorn Memorial Hospital. J Med Assoc Thai 2017;100:783-92.

11. Thewjitcharoen Y, Butadej S, Nakasatien S, et al. Incidence and malignancy rates classified by The Bethesda System for Reporting Thyroid Cytopathology (TBSRTC) - An 8-year tertiary center experience in Thailand. J Clin Transl Endocrinol 2018;16:100175.

12. Nakrangsee S. Cytopathology Reporting using the New Bethesda System of Thyroid FNAC and Correlation with Histopathological Follow-up: A Three-Year Study of Routine Service at Rajavithi Hospital. J Med Assoc Thai 2018;101:S122-32.

13. Sheffield BS, Masoudi H, Walker B, et al. Preoperative diagnosis of thyroid nodules using the Bethesda System for Reporting Thyroid Cytopathology: a comprehensive review and meta-analysis. Expert Rev Endocrinol Metab 2014;9:97-110.

14. Bongiovanni M, Spitale A, Faquin WC, et al. The Bethesda System for Reporting Thyroid Cytopathology: a meta-analysis. Acta Cytol 2012;56:333-9.

15. Krauss EA, Mahon M, Fede JM, et al. Application of the Bethesda Classification for Thyroid Fine-Needle Aspiration: Institutional Experience and Meta-analysis. Arch Pathol Lab Med 2016;140:1121-31.

16. Vuong HG, Ngo HTT, Bychkov A, et al. Differences in surgical resection rate and risk of malignancy in thyroid cytopathology practice between Western and Asian countries: A systematic review and meta-analysis. Cancer Cytopathol 2020;128:238-49.

17. Nikiforov YE, Biddinger PW, Thompson LDR. Diagnostic Pathology and Molecular Genetics of the Thyroid: A Comprehensive Guide for Practicing Thyroid Pathology. Lippincott Williams \& Wilkins (LWW); 2018: 480.

18. Anuwong A, Sasanakietkul T, Jitpratoom P, et al. Transoral endoscopic thyroidectomy vestibular approach (TOETVA): indications, techniques and results. Surg Endosc 2018;32:456-65.

19. Ban MJ, Chang JW, Kim WS, et al. Minimal Endoscopeassisted Thyroidectomy Through a Retroauricular Approach: An Evolving Solo Surgery Technique. Surg Laparosc Endosc Percutan Tech 2016;26:e109-12.

20. Byeon HK, Holsinger FC, Duvvuri U, et al. Recent progress of retroauricular robotic thyroidectomy with the new surgical robotic system. Laryngoscope 2018;128:1730-7.

21. Wisanuyothin T. Comparison of Endoscopic (Breastaxillary Approach) Versus Conventional Open Thyroid Lobectomy. The Thai Journal of Surgery. 2015;36(1).

22. Measuring overall health system performance for 191 countries. World Health Organization. Available online: https://www.who.int/healthinfo/paper30.pdf

23. Metter DM, Colgan TJ, Leung ST, et al. Trends in the US and Canadian Pathologist Workforces From 2007 to 2017. JAMA Netw Open 2019;2:e194337.

Cite this article as: Tangnuntachai N, Rangdaeng S, Koonmee S, Tangjaturonrasme N, Keelawat S. Pathological practice and management of thyroid nodules: a Thai perspective. Gland Surg 2020;9(5):1754-1763. doi: 10.21037/gs-20-395 